\title{
The thermal-statistical model for particle production
}

\author{
Jean Cleymans ${ }^{1, a}$
}

UCT-CERN Research Centre and Department of Physics, University of Cape Town, South Africa

\begin{abstract}
We introduce the thermal statistical model for particle production and its applications to heavy-ion collision experiments. We focus on the aspects related to chemical equilibrium, in particular on particle yields.
\end{abstract}

\section{Acknowledgment}

The author acknowledges the support of the JINR - South Africa cooperation agreement.

\section{References}

1. J. Cleymans and H. Satz, Zeitschrift für Physik C57, 135 (1993).

2. F. Becattini, J. Cleymans, A. Keränen, E. Suhonen and K. Redlich, Physical Review C64 (2001) 024901.

3. A. Andronic, P. Braun-Munzinger, J. Stachel, Nucl. Phys. A772, 167, 2006.

4. J. Cleymans, H. Oeschler and K. Redlich), Physical Review C59, (1999) 1663.

5. J. Cleymans, B. Kämpfer, M. Kaneta, S. Wheaton, N. Xu, Phys. Rev. C71, 0409071 (2005).

6. J. Cleymans, K. Redlich, Phys. Rev. Lett. 81, 5284 (1998).

7. C. Alt et al. (NA49 collaboration), Physical Review C77, 024903, 2008.

8. J. Cleymans, K. Redlich, Phys. Rev. C61, 054908 (1999).

9. F. Becattini, M. Gaździcki, J. Manninen, Phys. Rev. C73 (2006) 044905.

10. J. Takahashi (STAR collaboration) J. Phys. G36, 064074, 2009. J. Takahashi, STAR Collaboration.

11. V. Magas and H. Satz, Eur. Phys. J. C32 115 (2003).

12. P. Braun-Munzinger and J. Stachel, J. Phys. G:Nucl. Part. Phys. 281971 (2002).

13. J. Cleymans, H. Oeschler, K. Redlich, S. Wheaton Phys. Rev. C73, 034905 (2006).

14. J. Randrup and J.Cleymans, Phys. Rev. C74 (2006) 047901 .

15. J. Cleymans, R. Sahoo, D.P. Mahapatra, D.K. Srivastava, S. Wheaton, Physics Letters B660 (2008) 172-175.
16. J. Cleymans, B. Kämpfer, P. Steinberg and S. Wheaton, Journal of Physics G30 S595-S598 (2004).

17. J. Cleymans, H. Oeschler, K. Redlich and S. Wheaton, Physics Letters B615 (2005) 50-54.

18. P. Steinberg, J. Phys. G31 2005, S975.

19. A. Wroblewski, J. Phys. G31 2005, S621.

\footnotetext{
a e-mail: Jean.Cleymans@uct.ac.za
} 
The Thermal-Statistical Model for Particle Production I.

\author{
J. Cleymans
}

23 - 25 July 2008 / JINR, Dubna

\title{
Outline
}

Statistical Model

Strangeness

$E_{T} / N_{c h}$ vs. $E / N$

The Horn in the $K^{+} / \pi^{+}$Ratio 


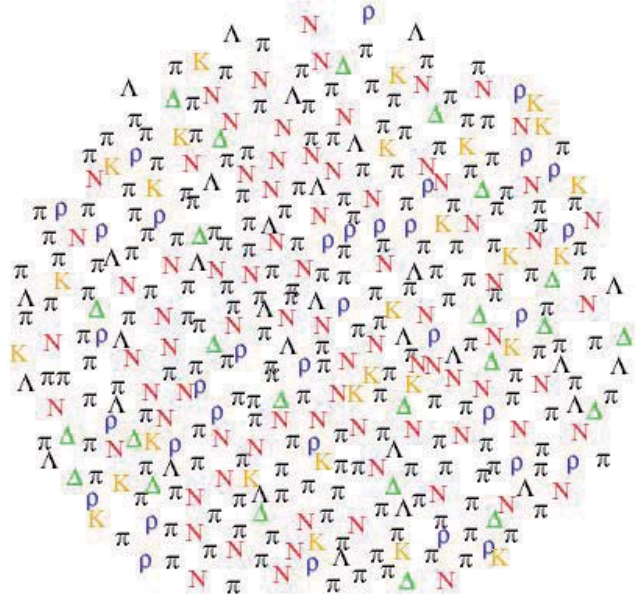

J.C. and H. Satz, Zeitschrift fuer Physik C57, 135 (1993)

\section{Thermal Equilibrium}

In thermal equilibrium

$$
Z=\operatorname{Tr} e^{-\frac{H}{T}+\frac{\mu N}{T}}
$$

$$
\langle N\rangle=\frac{\operatorname{Tr} N e^{-\frac{H}{T}+\frac{\mu N}{T}}}{\operatorname{Tr} e^{-\frac{H}{T}+\frac{\mu N}{T}}}
$$

$\langle E\rangle=\frac{\operatorname{Tr} E e^{\frac{-H}{T}+\frac{\mu N}{T}}}{\operatorname{Tr} e^{\frac{-H}{T}+\frac{\mu N}{T}}}$ 


\section{Full Hydrodynamic Flow}

Bjorken scaling + Transverse expansion

After integration over $m_{T}$

$$
\frac{d N_{i} / d y}{d N_{j} / d y}=\frac{N_{i}^{0}}{N_{j}^{0}}
$$

where $N_{i}^{0}$ is the particle yield as calculated in a fireball AT REST!

Effects of hydrodynamic flow cancel out in ratios.

\section{Thermal Equilibrium}

Particle Number

$$
\begin{aligned}
\langle N\rangle & =\frac{\operatorname{Tr} N e^{-\frac{H}{T}+\frac{\mu N}{T}}}{\operatorname{Tr} e^{-\frac{H}{T}+\frac{\mu N}{T}}} \\
& =\frac{T}{Z} \frac{\partial}{\partial \mu} \operatorname{Tr} e^{\frac{-H}{T}+\frac{\mu N}{T}} \\
& =T \frac{1}{Z} \frac{\partial Z}{\partial \mu} \\
& =T \frac{\partial}{\partial \mu} \ln Z
\end{aligned}
$$




\section{Thermal Equilibrium}

Average Energy

$$
\begin{aligned}
\langle E\rangle & =\frac{\operatorname{Tr} H e^{\frac{-H}{T}+\frac{\mu N}{T}}}{\operatorname{Tr} e^{\frac{-H}{T}+\frac{\mu N}{T}}} \\
& =-\frac{1}{Z} \frac{\partial Z}{\partial \beta}+\mu\langle N\rangle \\
& =T^{2} \frac{\partial}{\partial T} \ln Z+\mu\langle N\rangle
\end{aligned}
$$

\section{Thermal Equilibrium}

$$
\begin{aligned}
N_{i} & =g_{i} V \int \frac{d^{3} p}{(2 \pi)^{3}} \exp \left(-\frac{E}{T}\right) e^{\frac{\mu_{i}}{T}} \\
& =g_{i} V \frac{4 \pi}{(2 \pi)^{3}} \int p^{2} d p \exp \left(-\frac{\sqrt{p^{2}+m_{i}^{2}}}{T}\right) e^{\frac{\mu_{i}}{T}} \\
& =g_{i} V \frac{4 \pi}{(2 \pi)^{3}} T^{3} \int x^{2} d x \exp \left(-\sqrt{x^{2}+m_{i}^{2} / T^{2}}\right) e^{\frac{\mu_{i}}{T}} \\
& =g_{i} V \frac{1}{2 \pi^{2}} T m_{i}^{2} K_{2}\left(\frac{m_{i}}{T}\right) e^{\frac{\mu_{i}}{T}}
\end{aligned}
$$




\section{Thermal Equilibrium}

$$
\begin{aligned}
n_{i} & =g_{i} \frac{1}{2 \pi^{2}} T m_{i}^{2} K_{2}\left(\frac{m_{i}}{T}\right) e^{\frac{\mu_{i}}{T}} \\
\epsilon_{i} & =g_{i} \frac{1}{2 \pi^{2}} T m_{i}^{3}\left[K_{1}\left(\frac{m_{i}}{T}\right)+3 \frac{T}{m} K_{2}\left(\frac{m_{i}}{T}\right)\right] e^{\frac{\mu_{i}}{T}} \\
s_{i} & =g_{i} \frac{1}{2 \pi^{2}} m_{i}^{3}\left[K_{1}\left(\frac{m_{i}}{T}\right)+\frac{4 T}{m} K_{2}\left(\frac{m_{i}}{T}\right)-\frac{\mu_{i}}{m} K_{2}\left(\frac{m_{i}}{T}\right)\right] e^{\frac{\mu_{i}}{T}} \\
P_{i} & =g_{i} \frac{1}{2 \pi^{2}} T^{2} m_{i}^{2} K_{2}\left(\frac{m_{i}}{T}\right) e^{\frac{\mu_{i}}{T}}
\end{aligned}
$$

\section{Chemical Equilibrium}

In equilibrium

$$
E_{1}+E_{2}+\cdots=E_{3}+E_{4}+E_{5}+\cdots
$$

for the chemical potentials

$$
\mu_{1}+\mu_{2}+\cdots=\mu_{3}+\mu_{4}+\mu_{5}+\cdots
$$

As an example

$$
\pi^{0}+p \leftrightarrow \pi_{0}+p+\pi^{0}
$$

leads to

$$
\mu_{\pi^{0}}+\mu_{p}=\mu_{\pi^{0}}+\mu_{p}+\mu_{\pi^{0}}
$$

which leads to

$$
\mu_{\pi^{0}}=0
$$




\section{Chemical Equilibrium}

In equilibrium

$$
\begin{gathered}
B+B \leftrightarrow B+B+B+\bar{B} \\
d E=-p d V+T d S+\mu_{B} d N_{B}+\mu_{\bar{B}} d N_{\bar{B}}
\end{gathered}
$$

Due to baryon number conservation one has

$$
N_{B}-N_{\bar{B}}=\text { constant }
$$

and

$$
d N_{B}=d N_{\bar{B}}
$$

The energy is a minimum for

$$
\begin{gathered}
d E=\left(\mu_{B}+\mu_{\bar{B}}\right) d N_{B}=0 \\
\mu_{B}=-\mu_{\bar{B}}
\end{gathered}
$$

\section{Chemical Equilibrium}

In equilibrium

$$
\begin{gathered}
N_{B}=g \vee \int \frac{d^{3} p}{(2 \pi)^{3}} \exp \left(-\frac{E}{T}+\frac{\mu_{B}}{T}\right) \\
N_{\bar{B}}=g \vee \int \frac{d^{3} p}{(2 \pi)^{3}} \exp \left(-\frac{E}{T}-\frac{\mu_{B}}{T}\right) \\
N_{B}=N_{\bar{B}} \rightarrow \mu_{B}=0 \\
N_{B} \geq N_{\bar{B}} \rightarrow \mu_{B} \geq 0 \\
N_{B} \leq N_{\bar{B}} \rightarrow \mu_{B} \leq 0
\end{gathered}
$$




\begin{tabular}{|l|l|r|}
\hline & Chemical Equilibrium & No Chem. Equil. \\
\hline$\Lambda$ & $\exp \left[-\frac{E_{\pi}}{T}\right]$ & $\exp \left[-\frac{E_{\pi}}{T}+\frac{\mu_{\pi}}{T}\right]$ \\
$\bar{N}$ & $\exp \left[-\frac{E_{N}}{T}+\frac{\mu_{B}}{T}\right]$ & $\exp \left[-\frac{E_{N}}{T}+\frac{\mu_{N}}{T}\right]$ \\
$\Lambda$ & $\exp \left[-\frac{E_{N}}{T}-\frac{\underline{\mu}_{B}}{T}+\frac{\mu_{B}}{T}-\frac{\mu_{S}}{T}\right]$ & $\exp \left[-\frac{E_{\Lambda}}{T}+\frac{\mu_{\Lambda}}{T}\right]$ \\
$\bar{\Lambda}$ & $\exp \left[-\frac{E_{\Lambda}}{T}-\frac{\mu_{B}}{T}+\frac{\mu_{S}}{T}\right]$ & $\exp \left[-\frac{E_{\Lambda}}{T}+\frac{\mu_{\bar{K}}}{T}\right]$ \\
$K$ & $\exp \left[-\frac{E_{K}}{T}+\frac{\mu_{S}}{T}\right]$ & $\exp \left[-\frac{E_{K}}{T}+\frac{\mu_{K}}{T}\right]$ \\
$\bar{K}$ & $\exp \left[-\frac{E_{K}}{T}-\frac{\mu_{S}}{T}\right]$ & $\exp \left[-\frac{E_{K}}{T}+\frac{\mu_{K}}{T}\right]$
\end{tabular}

The number of particles of type $i$ is determined by:

$$
N_{i}=V g_{i} \int \frac{d^{3} p}{(2 \pi)^{3}} \exp \left(-\frac{E_{i}}{T}+\frac{\mu_{i}}{T}\right)
$$

For bosons:

$$
N_{i}=V g_{i} \int \frac{d^{3} p}{(2 \pi)^{3}} \frac{1}{\exp \left(\frac{E_{i}}{T}-\frac{\mu_{i}}{T}\right)-1}
$$

For fermions:

$$
N_{i}=V g_{i} \int \frac{d^{3} p}{(2 \pi)^{3}} \frac{1}{\exp \left(\frac{E_{i}}{T}-\frac{\mu_{i}}{T}\right)+1}
$$

Only conserved quantum numbers matter for chemical equilibrium: In equilibrium

$$
\mu_{i}=B_{i} \mu_{B}+Q_{i} \mu_{Q}+S_{i} \mu_{S}+C_{i} \mu_{C}+.
$$




\begin{tabular}{|c|c|c|c|c|c|c|}
\hline$g_{i}$ & $m_{i}$ & stat & $S_{i}$ & $B_{i}$ & $Q_{i}$ & Particle $i$ \\
\hline & & & & & & \\
1 & 0.140 & -1 & 0 & 0 & 1. & $\pi^{+}$ \\
1 & 0.135 & -1 & 0 & 0 & 0. & $\pi^{0}$ \\
1 & 0.140 & -1 & 0 & 0 & -1. & $\pi^{-}$ \\
1 & 0.547 & -1 & 0 & 0 & 0. & $\eta$ \\
3 & 0.770 & -1 & 0 & 0 & 1. & $\rho^{+}$ \\
3 & 0.770 & -1 & 0 & 0 & 0. & $\rho^{0}$ \\
3 & 0.770 & -1 & 0 & 0 & -1. & $\rho^{-}$ \\
3 & 0.782 & -1 & 0 & 0 & 0. & $\omega$ \\
1 & 0.958 & -1 & 0 & 0 & 0. & $\eta^{\prime}$ \\
1 & 0.980 & -1 & 0 & 0 & 0. & $f_{0}$ \\
1 & 0.982 & -1 & 0 & 0 & 1. & $a_{0}^{+}$ \\
1 & 0.982 & -1 & 0 & 0 & 0. & $a_{0}^{0}$ \\
1 & 0.982 & -1 & 0 & 0 & -1. & $a_{0}^{-}$ \\
3 & 1.019 & -1 & 0 & 0 & 0. & $\phi$ \\
3 & 1.170 & -1 & 0 & 0 & 0. & \\
3 & 1.230 & -1 & 0 & 0 & 1. & \\
3 & 1.230 & -1 & 0 & 0 & 0. & \\
3 & 1.230 & -1 & 0 & 0 & -1. & \\
3 & 1.229 & -1 & 0 & 0 & 1. & \\
3 & 1.229 & -1 & 0 & 0 & 0. & \\
3 & 1.229 & -1 & 0 & 0 & -1. & \\
5 & 1.275 & -1 & 0 & 0 & 0. & \\
3 & 1.282 & -1 & 0 & 0 & 0. & \\
1 & 1.297 & -1 & 0 & 0 & 0. & \\
1 & 1.300 & -1 & 0 & 0 & 1. & \\
1 & 1.300 & -1 & 0 & 0 & 0. & \\
\hline & & & & & & \\
\hline
\end{tabular}

\section{The Role of Resonances}

Example: $\rho$ 's

$$
\rho \rightarrow \pi^{+} \pi^{-}
$$

Final, observed, number of $\pi^{+}$is given by

$$
N_{\pi^{+}}=N_{\pi^{+}}(\text {thermal })+N_{\pi^{+}}(\text {resonance decays })
$$

depending on the temperature, over $80 \%$ of observed pions are due to resonance decays 
Importance of Resonances.

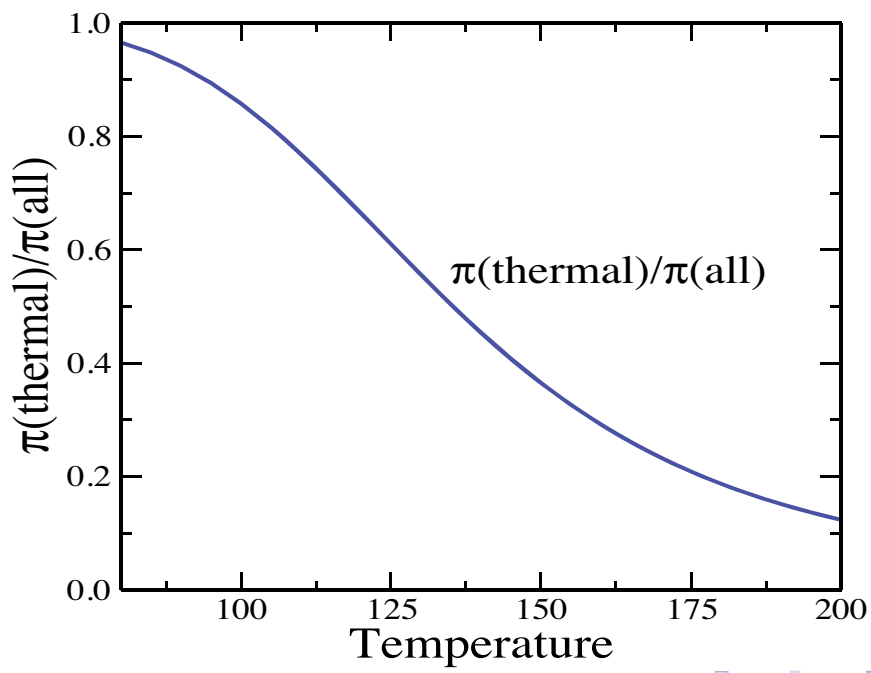

Strangeness saturation?

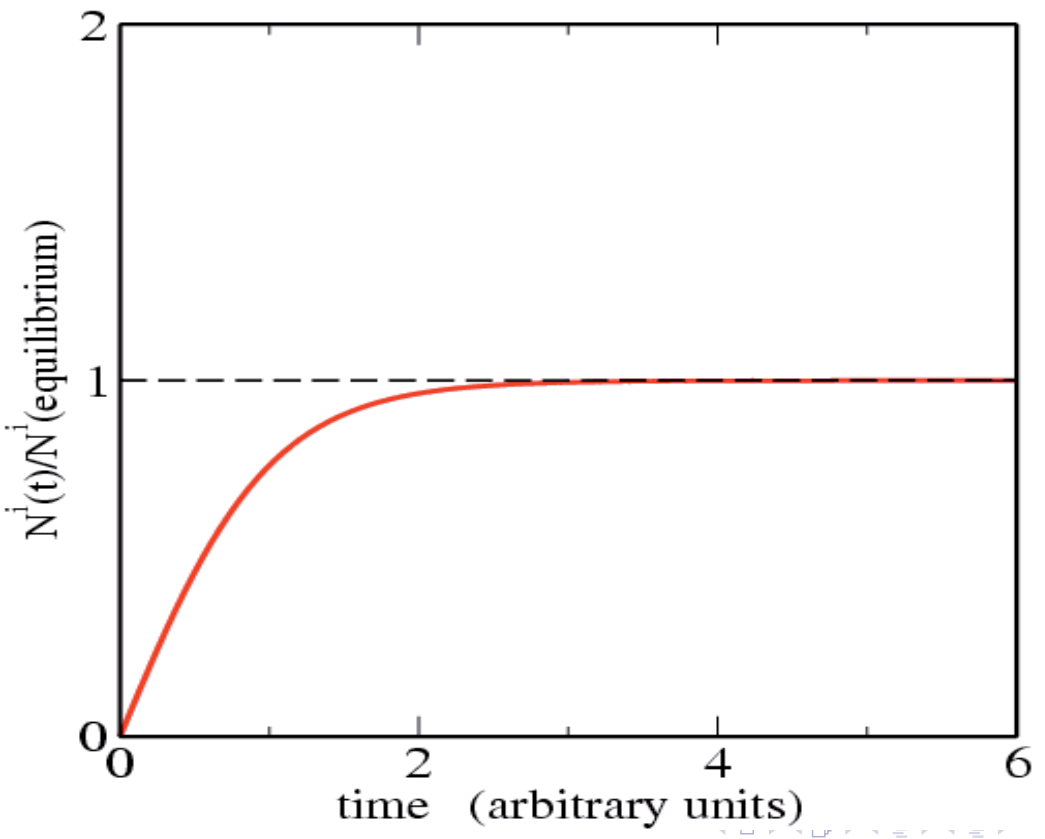




\section{Strangeness saturation?}

$$
N_{i}=\gamma_{s}^{|S|} V g_{i} \int \frac{d^{3} p}{(2 \pi)^{3}} \exp \left(-\frac{E_{i}}{T}+\frac{\mu_{i}}{T}\right)
$$

with

$\gamma_{s}<1$ strangeness under-saturation

$\gamma_{s}=1$ strangeness in chemical equilibrium

$\gamma_{s}>1$ strangeness over-saturation

SPS data.

\begin{tabular}{|c|c|}
\hline & Measurement \\
\hline \multicolumn{2}{|c|}{$\mathrm{Pb}-\mathrm{Pb} 158 A \mathrm{GeV}$} \\
\hline$\left(\pi^{+}+\pi^{-}\right) / 2$. & $600 \pm 30$ \\
$\mathrm{~K}^{+}$ & $95 \pm 10$ \\
$\mathrm{~K}^{-}$ & $50 \pm 5$ \\
$\mathrm{~K}_{S}^{0}$ & $60 \pm 12$ \\
$\mathrm{p}$ & $140 \pm 12$ \\
$\overline{\mathrm{p}}$ & $10 \pm 1.7$ \\
$\phi$ & $7.6 \pm 1.1$ \\
$\bar{\Xi}^{-}$ & $4.42 \pm 0.31$ \\
$\bar{\Xi}^{-}$ & $0.74 \pm 0.04$ \\
$\bar{\Lambda} / \Lambda$ & $0.2 \pm 0.04$ \\
\hline
\end{tabular}




\section{SPS data.}

SPS: Chemical Freeze-Out Parameters:

$$
\begin{aligned}
T & =156.0 \pm 2.4 \mathrm{MeV} \\
\mu_{B} & =239 \pm 12 \mathrm{MeV} \\
\gamma_{s} & =0.862 \pm 0.036
\end{aligned}
$$

F. Becattini, J.C., A. Keränen, E. Suhonen and K. Redlich Physical Review C64 (2001) 024901.

\section{AGS data.}

\begin{tabular}{|c|c|}
\hline & Measurement \\
\hline \multicolumn{2}{|c|}{ Au-Au 11.6A GeV } \\
\hline Participants & $363 \pm 10$ \\
$\mathrm{~K}^{+}$ & $23.7 \pm 2.9$ \\
$\mathrm{~K}^{-}$ & $3.76 \pm 0.47$ \\
$\pi^{+}$ & $133.7 \pm 9.9$ \\
$\Lambda$ & $20.34 \pm 2.74$ \\
$\mathrm{p} / \pi^{+}$ & $1.234 \pm 0.126$ \\
\hline$\overline{\mathrm{p}}$ & $>0.0185 \pm 0.0018$ \\
\hline
\end{tabular}




\section{AGS data.}

AGS: Chemical Freeze-Out Parameters:

$$
\begin{aligned}
T & =130.6 \pm 5.5 \mathrm{MeV} \\
\mu_{B} & =594 \pm 26 \mathrm{MeV} \\
\gamma_{s} & =0.883 \pm 0.124
\end{aligned}
$$

F. Becattini, J.C., A. Keränen, E. Suhonen and K. Redlich Physical Review C64 (2001) 024901.

\section{SIS data.}

\begin{tabular}{|c|c|}
\hline & Measurement \\
\hline \multicolumn{2}{|c|}{$\mathrm{Au}-\mathrm{Au} \mathrm{1.7A} \mathrm{GeV}$} \\
\hline$\pi^{+} / \mathrm{p}$ & $0.052 \pm 0.013$ \\
$\mathrm{~K}^{+} / \pi^{+}$ & $0.003 \pm 0.00075$ \\
$\pi^{-} / \pi^{+}$ & $2.05 \pm 0.51$ \\
$\eta / \pi^{0}$ & $0.018 \pm 0.007$ \\
\hline
\end{tabular}

SIS: Chemical Freeze-Out Parameters:

$$
\begin{aligned}
T & =49.7 \pm 1.1 \mathrm{MeV} \\
\mu_{B} & =818 \pm 15 \mathrm{MeV} \\
\gamma_{s} & =1 \text { (fixed) }
\end{aligned}
$$

J. C., H. Oeschler and K. Redlich)

Physical Review C59, (1999) 1663. 
EPJ Web of Conferences

\section{RHIC data.}

J. C., B. Kämpfer, M. Kaneta, S. Wheaton, N. Xu, Phys. Rev. C71, 0409071 (2005)

\begin{tabular}{|c|c|c|c|c|}
\hline Ratio & Experiment & Central & Mid-Central & Peripheral \\
\hline \multirow{4}{*}{$\pi_{(2)}^{-} / \pi_{(2)}^{+}$} & BRAHMS & $0.990 \pm 0.100$ & & \\
\hline & PHENIX & $0.960 \pm 0.177$ & $0.920 \pm 0.170$ & $0.933 \pm 0.172$ \\
\hline & PHOBOS & $1.000 \pm 0.022$ & & \\
\hline & STAR & $1.000 \pm 0.073$ & $1.000 \pm 0.073$ & $1.000 \pm 0.073$ \\
\hline \multirow{3}{*}{$K_{(2)}^{+} / K_{(2)}^{-}$} & PHENIX & $1.152 \pm 0.240$ & $1.292 \pm 0.268$ & $1.322 \pm 0.284$ \\
\hline & PHOBOS & $1.099 \pm 0.111$ & & \\
\hline & STAR & $1.109 \pm 0.022$ & $1.105 \pm 0.036$ & $1.120 \pm 0.040$ \\
\hline $\bar{p}_{(1)} / p_{(1)}$ & PHENIX & $0.680 \pm 0.149$ & $0.671 \pm 0.142$ & $0.717 \pm 0.157$ \\
\hline \multirow[t]{3}{*}{$\bar{p}_{(2)} / p_{(2)}$} & BRAHMS & $0.650 \pm 0.092$ & & \\
\hline & PHOBOS & $0.600 \pm 0.072$ & & \\
\hline & STAR & $0.714 \pm 0.050$ & $0.724 \pm 0.050$ & $0.764 \pm 0.053$ \\
\hline $\bar{\Lambda}_{(1)} / \Lambda_{(1)}$ & PHENIX & $0.750 \pm 0.180$ & $0.798 \pm 0.197$ & $0.795 \pm 0.197$ \\
\hline $\bar{\Lambda}_{(2)} / \Lambda_{(2)}$ & STAR & $0.719 \pm 0.090$ & $0.739 \pm 0.092$ & $0.744 \pm 0.100$ \\
\hline $\bar{\Xi}_{(2)}^{+} / \Xi_{(2)}^{-}$ & STAR & $0.840 \pm 0.053$ & $0.822 \pm 0.114$ & $0.815 \pm 0.096$ \\
\hline $\bar{\Omega}^{+} / \Omega^{-}$ & STAR & $1.062 \pm 0.410$ & & \\
\hline \multirow[t]{2}{*}{$K_{(2)}^{-} / \pi_{(2)}^{-}$} & PHENIX & $0.151 \pm 0.030$ & $0.134 \pm 0.027$ & $0.116 \pm 0.023$ \\
\hline & STAR & $0.151 \pm 0.022$ & $0.147 \pm 0.022$ & $0.130 \pm 0.019$ \\
\hline$K_{S}^{0} / \pi_{(2)}^{-}$ & STAR & $0.134 \pm 0.022$ & $0.131 \pm 0.022$ & $0.108 \pm 0.018$ \\
\hline $\bar{p}_{(1)} / \pi_{(2)}^{-}$ & PHENIX & $0.049 \pm 0.010$ & $0.047 \pm 0.010$ & $0.045 \pm 0.009$ \\
\hline $\bar{p}_{(2)} / \pi_{(2)}$ & STAR & $0.069 \pm 0.019$ & $0.067 \pm 0.019$ & $0.067 \pm 0.019$ \\
\hline$\Lambda_{(1)} / \pi_{(2)}$ & STAR & $0.043 \pm 0.008$ & $0.043 \pm 0.008$ & $0.039 \pm 0.007$ \\
\hline$\Lambda_{(2)} / \pi_{(2)}$ & PHENIX & $0.072 \pm 0.017$ & $0.068 \pm 0.016$ & $0.074 \pm 0.017$ \\
\hline$<K^{* 0}>/ \pi_{(2)}^{-}$ & STAR & $0.039 \pm 0.011$ & & \\
\hline$\phi / \pi_{(2)}^{-}$ & STAR & $0.022 \pm 0.003$ & $0.021 \pm 0.004$ & $0.022 \pm 0.004$ \\
\hline$\Xi_{(n)}^{-} / \pi / \pi$ & STAR & $0.0093 \pm 0.0012$ & $0.0072 \pm 0.0011$ & $0.0060 \pm 0.0008$ \\
\hline
\end{tabular}

\section{RHIC data.}

RHIC: Chemical Freeze-Out Parameters:

$$
\begin{aligned}
T & =169 \pm 4.2 \mathrm{MeV} \\
\mu_{B} & =39.6 \pm 6 \mathrm{MeV} \\
\gamma_{s} & =0.9 \pm 0.1
\end{aligned}
$$

J. C., B. Kämpfer, M. Kaneta, S. Wheaton, N. Xu

Phys. Rev. C71, 0409071 (2005) 


\section{$\mathrm{E} / \mathrm{N}$ in 1999}

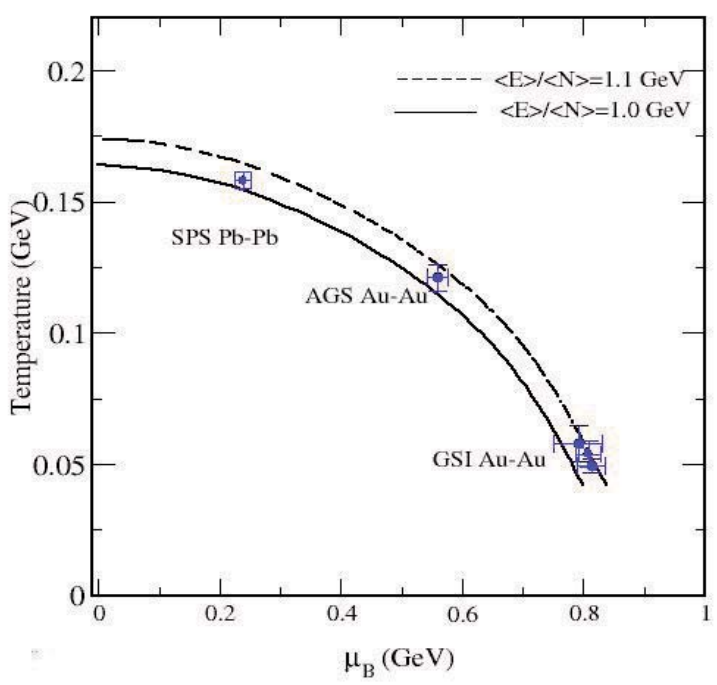

\section{$\mathrm{E} / \mathrm{N}$ in 2000}

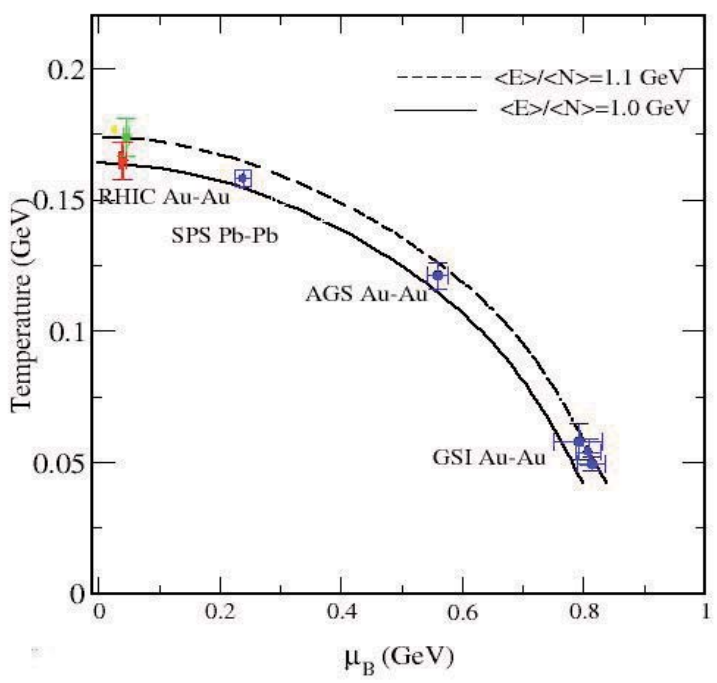


EPJ Web of Conferences

$\mathrm{E} / \mathrm{N}$ in 2005
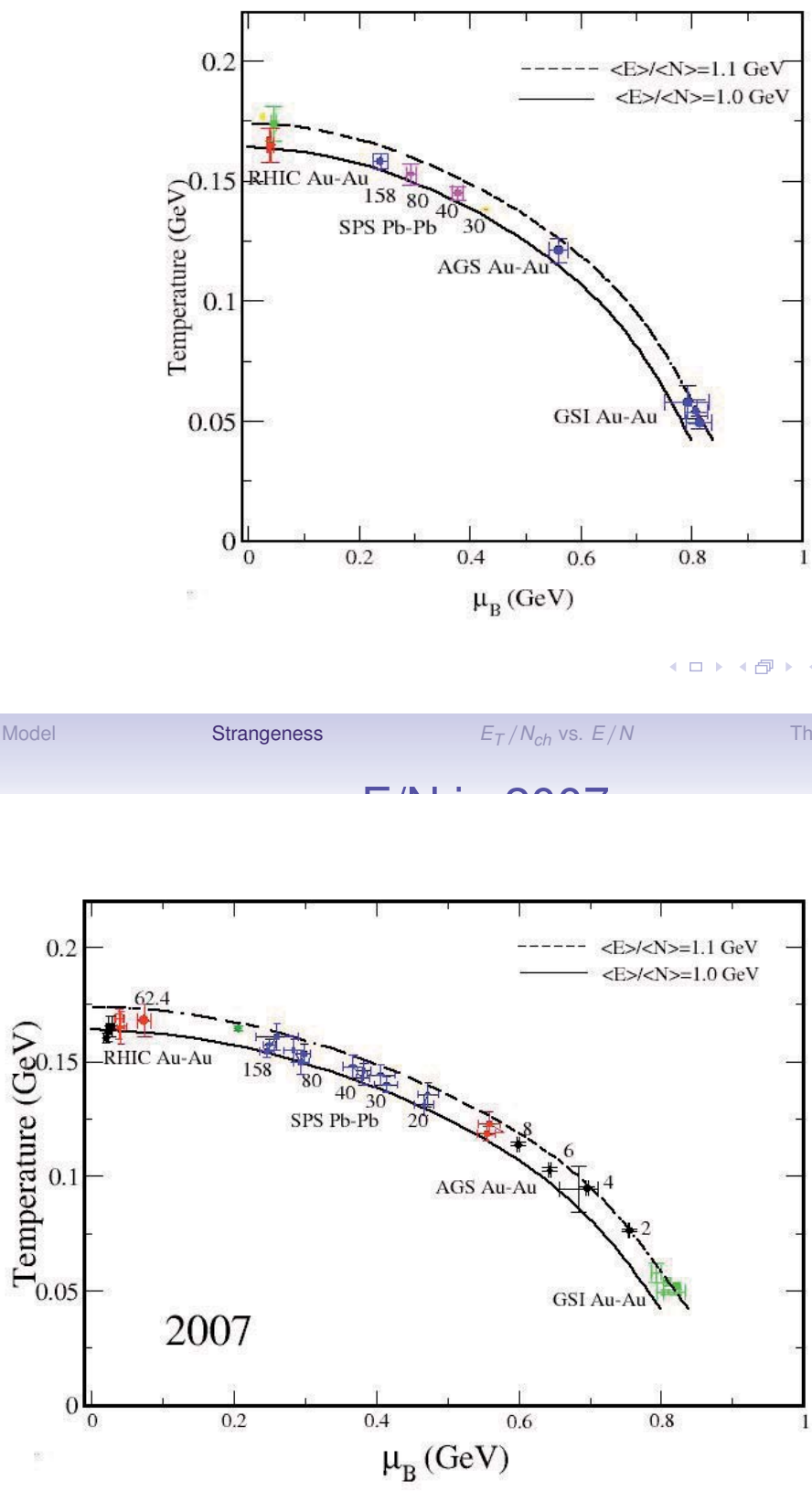


\section{Chemical Freeze-Out: Status in 2005}

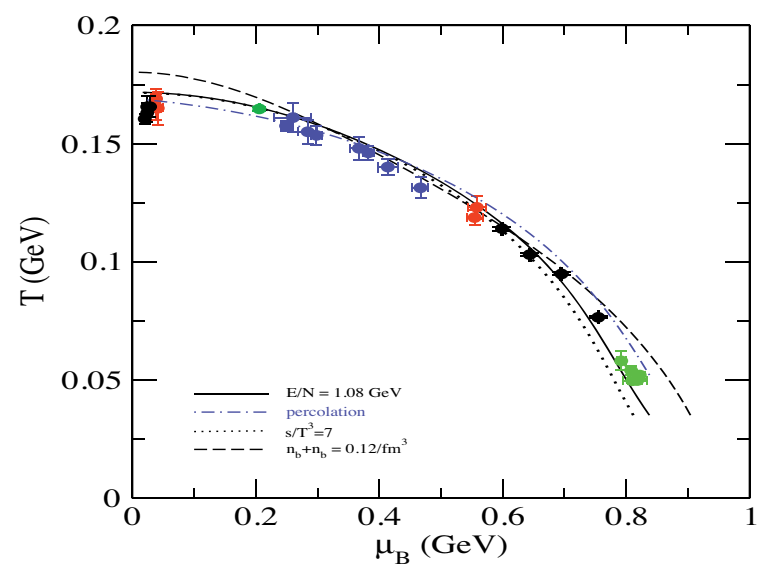

V. Magas and H. Satz, Eur. Phys. J. C32 115 (2003).

P. Braun-Munzinger and J. Stachel, J. Phys. G:Nucl. Part. Phys. 281971 (2002).

\section{Chemical Freeze-Out: Status in 2005}

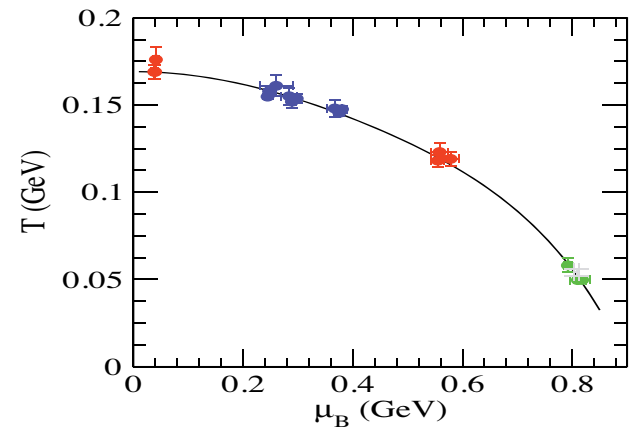

$$
T\left(\mu_{B}\right)=0.169-0.189 \mu_{B}^{2}+0.165 \mu_{B}^{4}-0.229 \mu_{B}^{6} .
$$

J. C., H. Oeschler, K. Redlich, S. Wheaton 


\section{$\mu_{B}$ as a function of $\sqrt{s_{N N}}$}

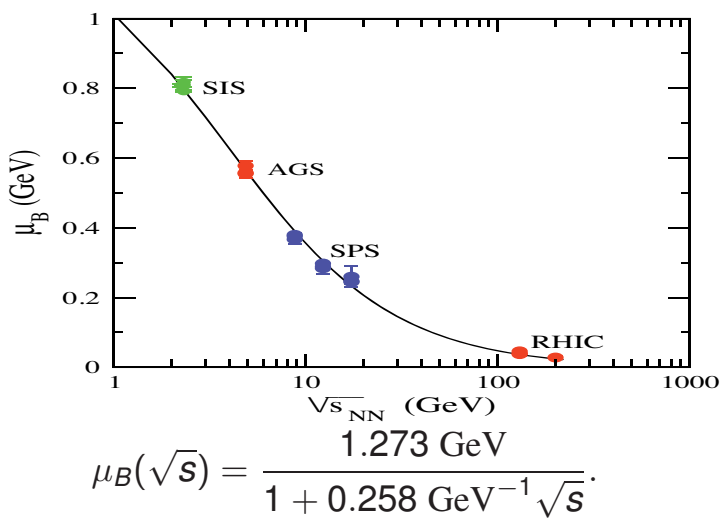

This predicts at $\mathrm{LHC} \mu_{B} \approx 1 \mathrm{MeV}$.

J. C., H. Oeschler, K. Redlich, S. Wheaton

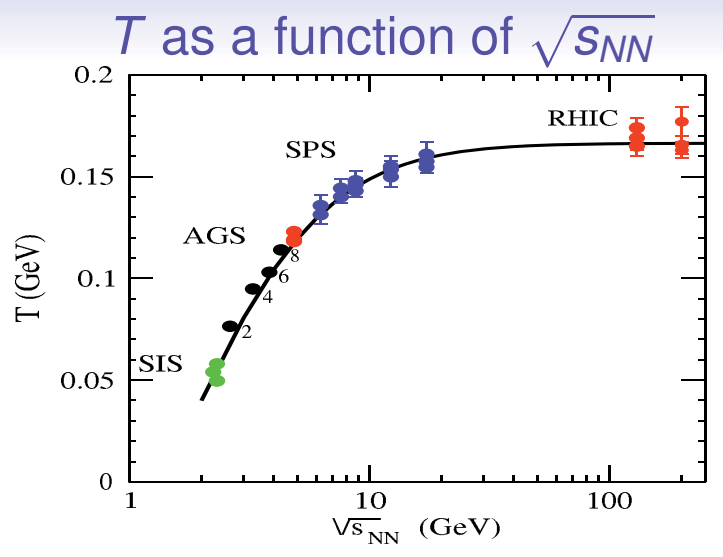

This predicts at $\mathrm{LHC} T \approx 170 \mathrm{MeV}$.

J. C., H. Oeschler, K. Redlich, S. Wheaton 


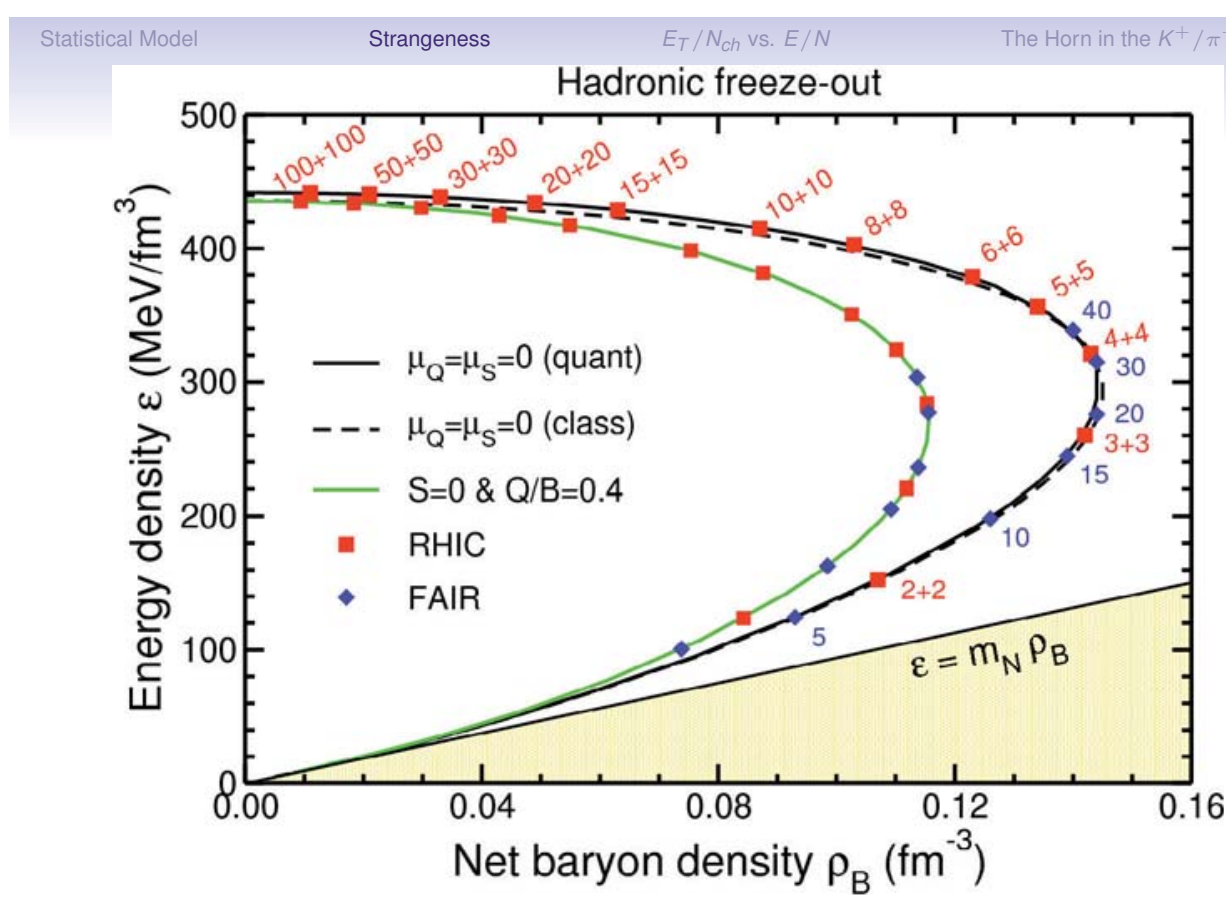

J. Randrup and J.C., Phys. Rev. C74 (2006) 047901

Will it be possible to determine directly $\mathrm{E} / \mathrm{N}$ ?

$E$ : energy of primordial hadrons

$N$ : number of primordial hadrons

$$
\begin{aligned}
\left\langle E_{T}\right\rangle & =\langle E \sin \theta\rangle \\
& =\frac{\pi}{4}\langle E\rangle
\end{aligned}
$$

Low energy limit

$$
\lim \frac{E_{T}}{N_{\mathrm{ch}}}=\frac{\frac{\pi}{4} m_{N}}{0.4} \approx 1.8 \mathrm{GeV}
$$

High energy limit

$$
\lim \frac{E_{T}}{N_{\mathrm{ch}}}=\frac{\frac{\pi}{4}\langle M\rangle}{2 / 3} \approx 0.9 \mathrm{GeV}
$$

However $E_{T}$ : subtract $m_{N}$ for baryons add $m_{N}$ for antibaryons. 
Primordial vs Final State Hadrons

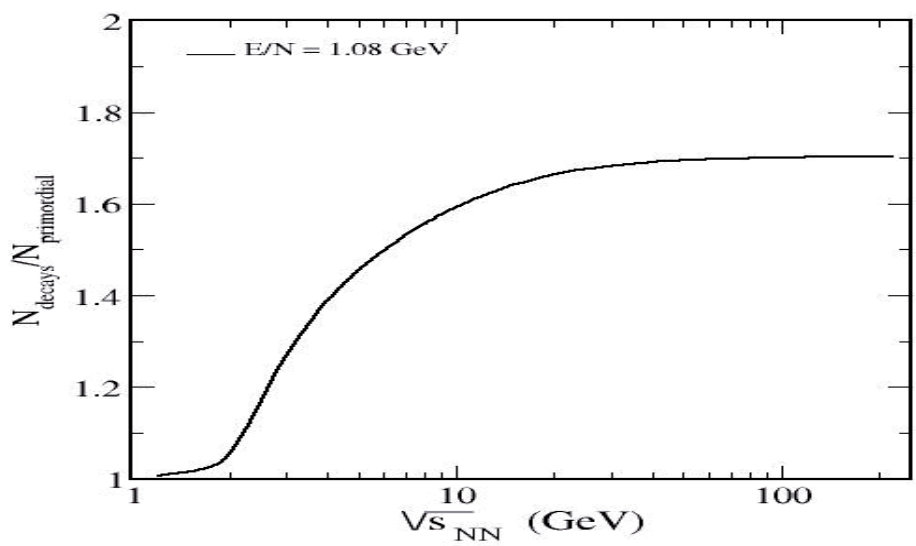

J.C., R. Sahoo, D.K. Srivastava, S. Wheaton

Average Mass in Fireball

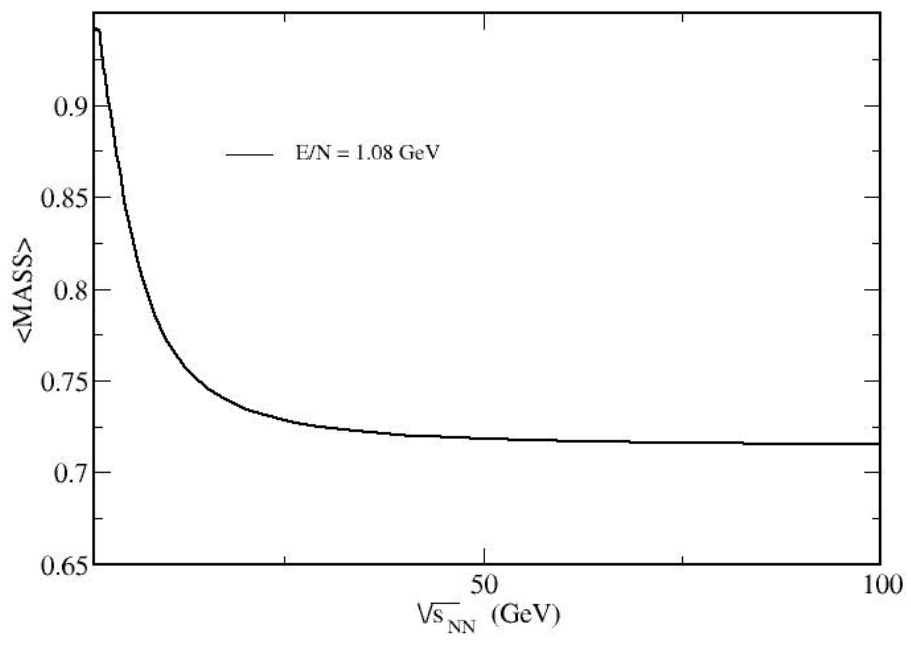

J.C., R. Sahoo, D.K. Srivastava, S. Wheaton 
Transverse Energy per Charge

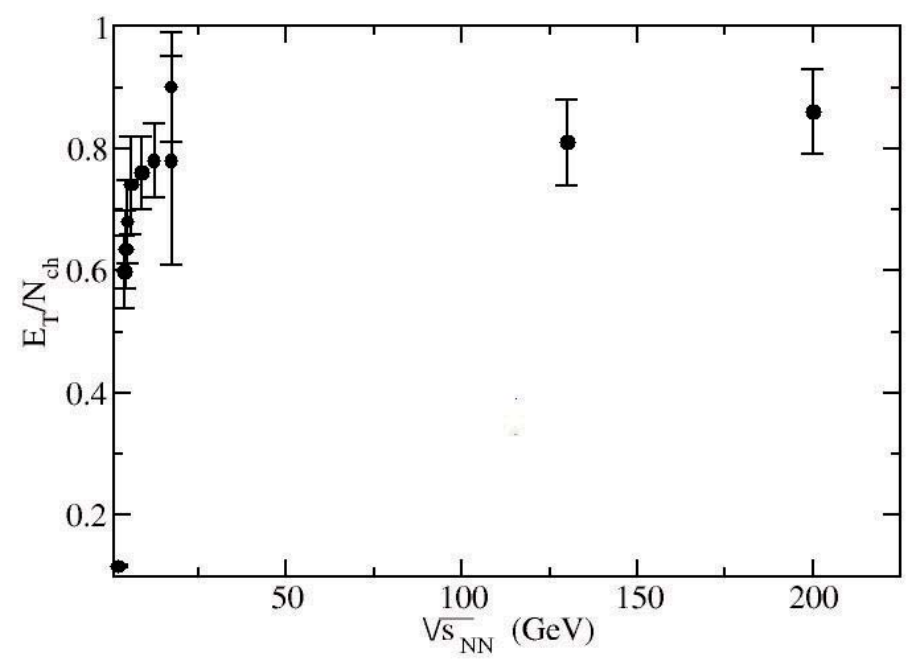

Transverse Energy per Charged Hadron

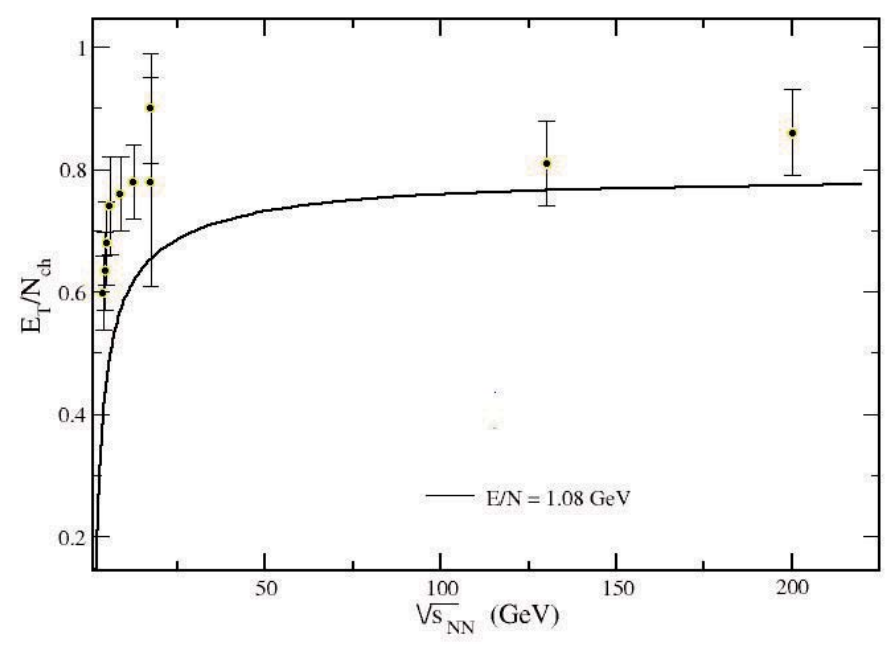

J.C., R. Sahoo, D.K. Srivastava, S. Wheaton 
EPJ Web of Conferences

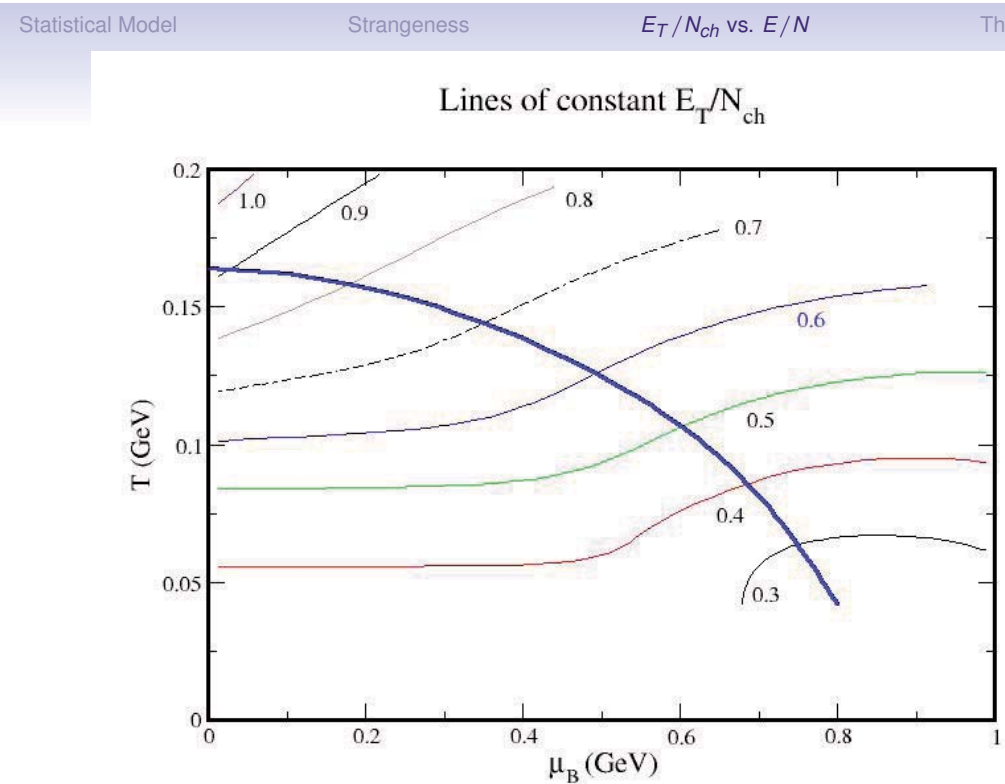

mainly follows $T$ and is determined by $E / N$,

J.C., R. Sahoo, D.K. Srivastava, S. Wheaton

Centrality Dependence of the Baryon Chemical

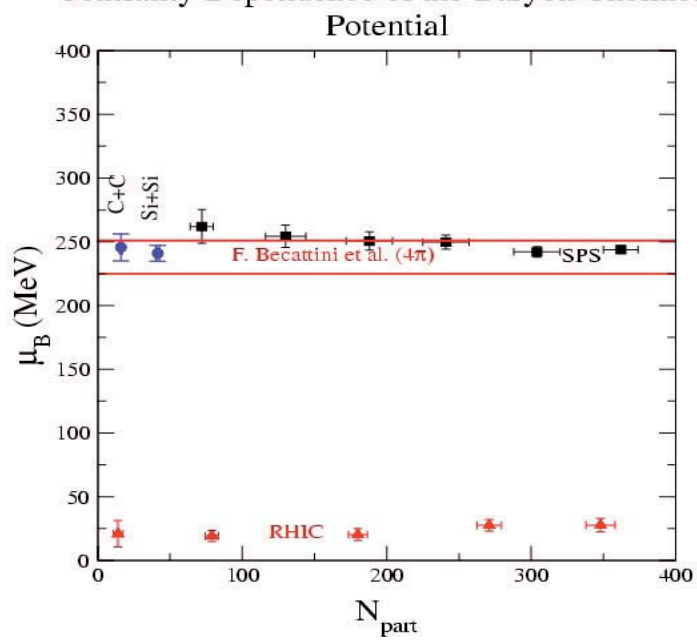

J. C., B. Kämpfer, P. Steinberg and S. Wheaton, Journal of Physics G30 S595-S598 (2004). 
Dense Matter In Heavy Ion Collisions and Astrophysics (DM2008)

\section{Centrality Dependence of the Chemical Freeze-out} Temperature

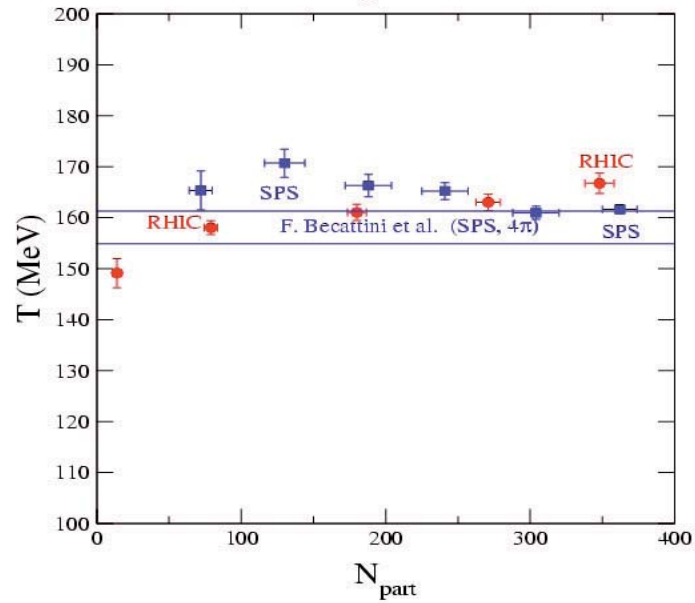

J. C., B. Kämpfer, P. Steinberg and S. Wheaton, Journal of Physics G30 S595-S598 (2004).

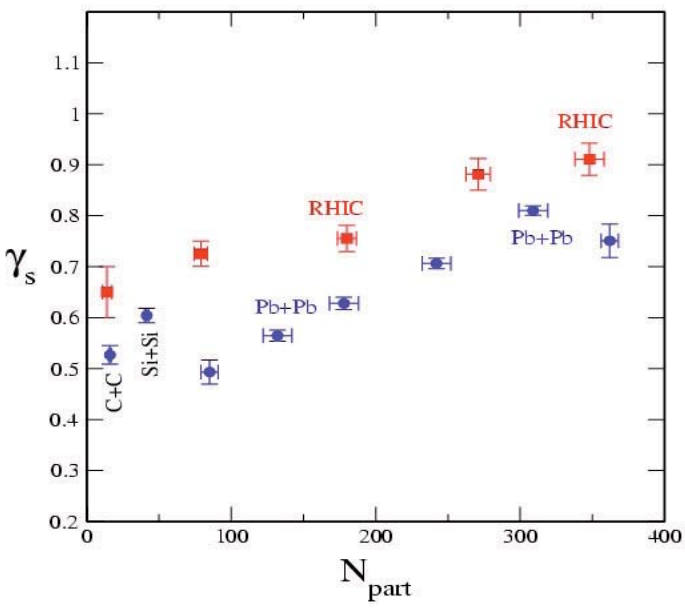

J. C., B. Kämpfer, P. Steinberg and S. Wheaton, Journal of Physics G30 S595-S598 (2004). 
The NA49 Collaboration has recently performed a series of measurements of $\mathrm{Pb}-\mathrm{Pb}$ collisions at 20, 30, 40, 80 and 158 $A G e V$ beam energies. When these results are combined with measurements at lower beam energies from the AGS they reveal an unusually sharp variation with beam energy in the $\Lambda /\langle\pi\rangle$, with $\langle\pi\rangle \equiv 3 / 2\left(\pi^{+}+\pi^{-}\right)$, and $K^{+} / \pi^{+}$ratios. Such a strong variation with energy does not occur in pp collisions and therefore indicates a major difference in heavy-ion collisions. This transition has been referred as the "horn".

\section{The Elephant in the Room}

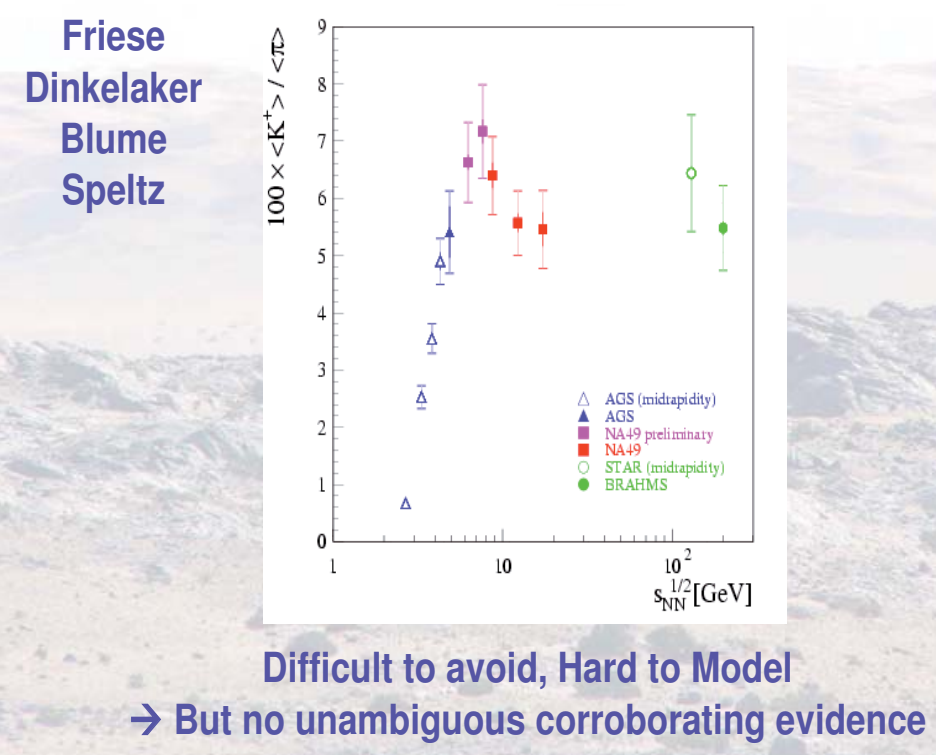




\section{Strangeness in Heavy lon Collisions}

vs

\section{Strangeness in pp - collisions}

Use the Wroblewski factor

$$
\lambda_{s}=\frac{2\langle s \bar{s}\rangle}{\langle u \bar{u}\rangle+\langle d \bar{d}\rangle}
$$

This is determined by the number of newly created quark anti-quark pairs and before strong decays, i.e. before $\rho$ 's and $\Delta$ 's decay.

Limiting values :

$\lambda_{s}=1$ all quark pairs are equally abundant, $\mathrm{SU}(3)$ symmetry. $\lambda_{s}=0$ no strange quark pairs.

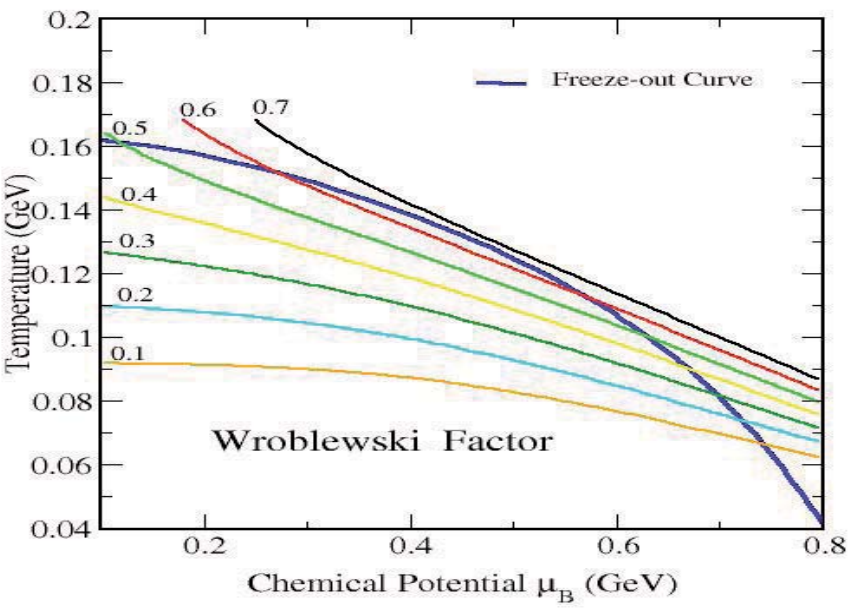


EPJ Web of Conferences

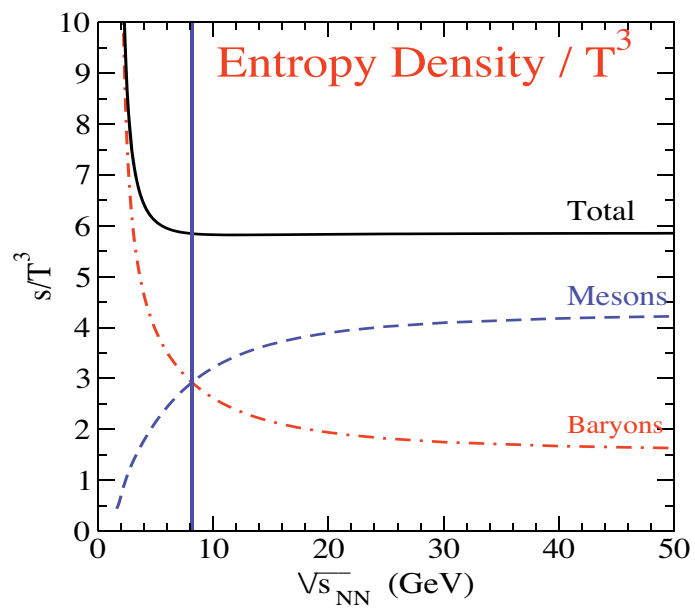

J. C., H. Oeschler, K. Redlich and S. Wheaton, Physics Letters B615 (2005) 50-54

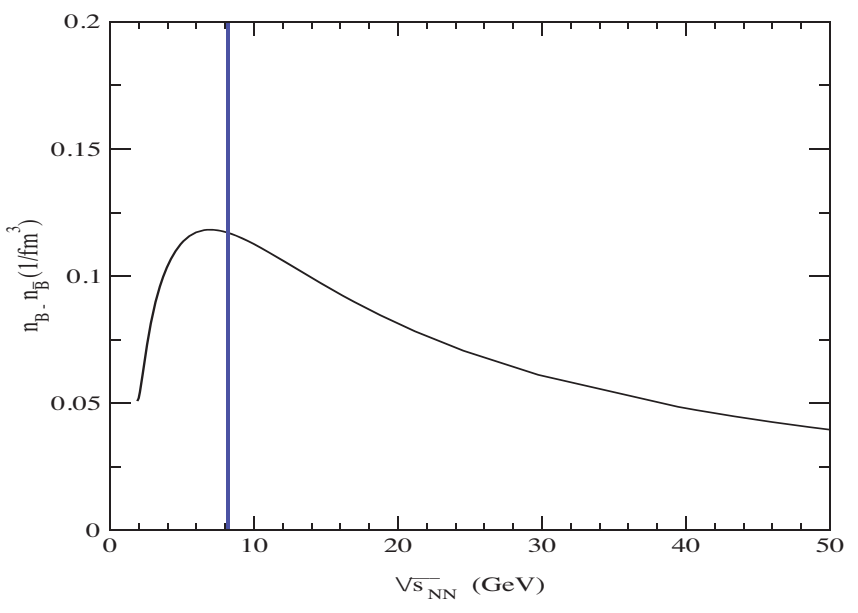

J. C., H. Oeschler, K. Redlich and S. Wheaton, Physics Letters B615 (2005) 50-54. 
Dense Matter In Heavy Ion Collisions and Astrophysics (DM2008)

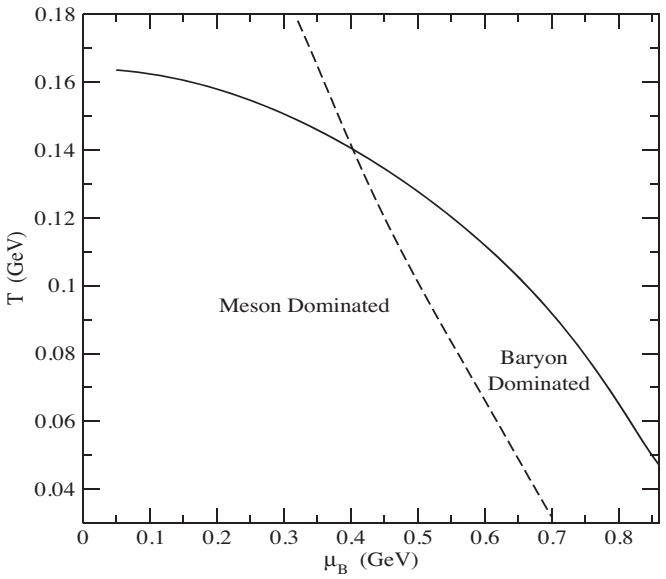

J. C., H. Oeschler, K. Redlich and S. Wheaton, Physics Letters B615 (2005) 50-54.

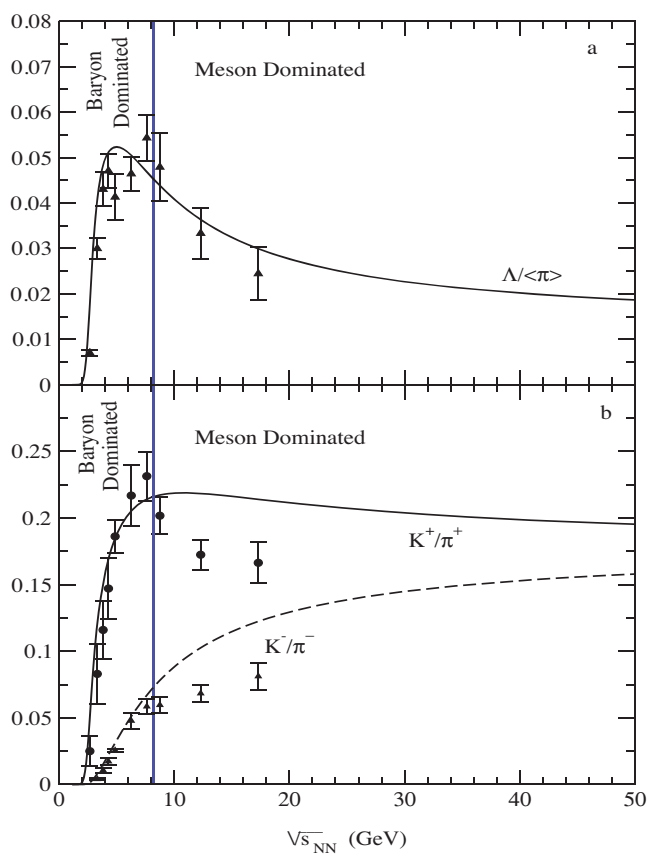

J. C., H. Oeschler, K. Redlich and S. Wheaton, Physics Letters B615 (2005) 50-54 


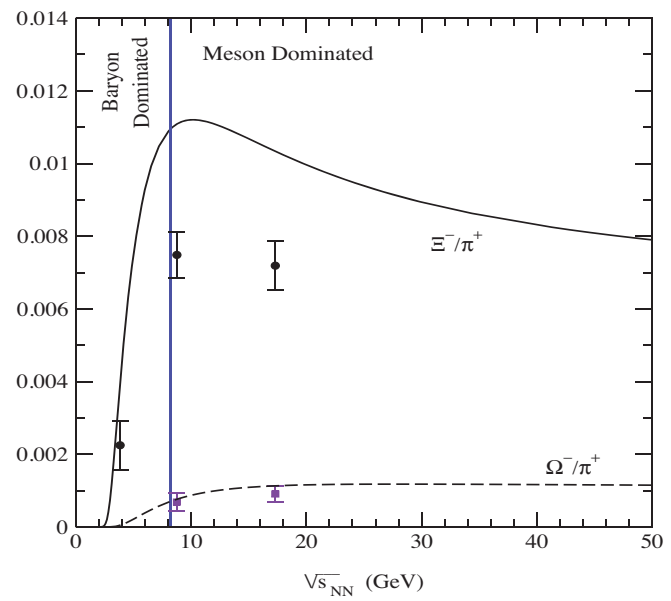

J. C., H. Oeschler, K. Redlich and S. Wheaton, Physics Letters B615 (2005) 50-54

Maxima in Particle Ratios predicted by the Thermal Model.

\begin{tabular}{|l|c|c|}
\hline Ratio & $\begin{array}{c}\text { Maximum at } \\
\sqrt{s_{N N}}(\mathrm{GeV})\end{array}$ & $\begin{array}{c}\text { Maximum } \\
\text { Value }\end{array}$ \\
\hline$\Lambda /\langle\pi\rangle$ & 5.1 & 0.052 \\
$\Xi^{-} / \pi^{+}$ & 10.2 & 0.011 \\
$K^{+} / \pi^{+}$ & 10.8 & 0.22 \\
$\Omega^{-} / \pi^{+}$ & 27 & 0.0012 \\
\hline
\end{tabular}

J. C., H. Oeschler, K. Redlich and S. Wheaton, Physics Letters B615 (2005) 50-54. 


\title{
The Thermal-Statistical Model for Particle Production II.
}

\author{
J. Cleymans
}

25 July 2008 / Dubna, Russia

\section{Outline}

Hydrodynamic Flow

Excluded Volume Corrections

Canonical Corrections

Transverse Momentum Distributions

Rapidity Distributions 


\section{Hydrodynamic Flow.}

\section{Cooper-Frye formula}

From

$$
N=\int j^{\mu} d \sigma_{\mu} \text { and } j^{\mu}=\int d^{3} p \frac{p^{\mu}}{E} f(r, p, t)
$$

obtain

$$
E \frac{d N}{d^{3} p}=\int p^{\mu} d \sigma_{\mu} f(r, p, t)
$$

The number of particles of type $i$ is determined by:

$$
E \frac{d N_{i}}{d^{3} p}=\frac{g_{i}}{(2 \pi)^{3}} \int d \sigma_{\mu} p^{\mu} \exp \left(-\frac{p^{\mu} u_{\mu}}{T}+\frac{\mu_{i}}{T}\right)
$$

Integrating this over all momenta

$$
N_{i}=\frac{g_{i}}{(2 \pi)^{3}} \int d \sigma_{\mu} \int \frac{d^{3} p}{E} p^{\mu} \exp \left(-\frac{p^{\mu} u_{\mu}}{T}+\frac{\mu_{i}}{T}\right)
$$

or

$$
N_{i}=\int d \sigma_{\mu} u^{\mu} n_{i}(T, \mu)
$$

If the temperature and chemical potential are unique along the freeze-out curve

$$
\begin{aligned}
& \text { Particle Yield: } \\
& N_{i}=n_{i}(T, \mu) \int d \sigma_{\mu} u^{\mu}
\end{aligned}
$$

i.e. integrated $(4 \pi)$ multiplicities are the same as for a single fireball at rest (apart from the volume). 
Dense Matter In Heavy Ion Collisions and Astrophysics (DM2008)

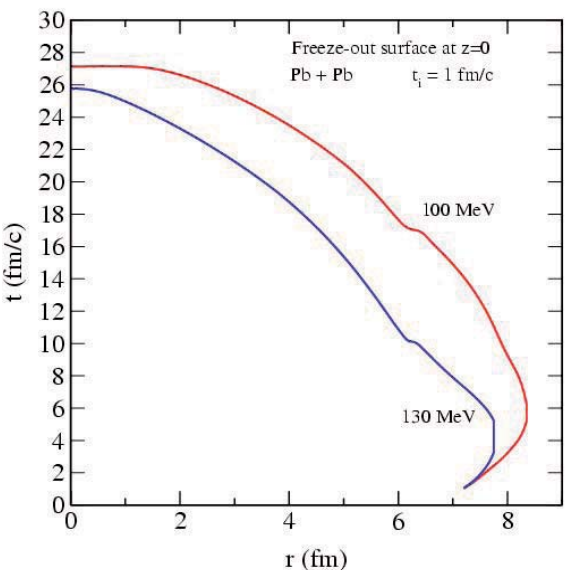

J. Cleymans, K. Redlich, D.K. Srivastava

Phys. Rev. C55 (1997) 1431

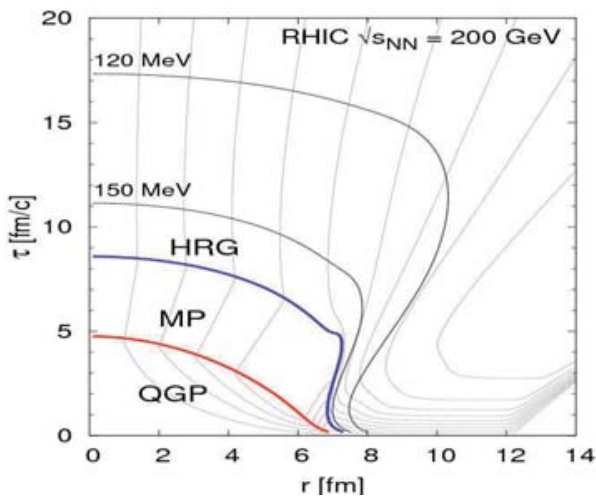

K. Eskola, H. Honkanen, H.Niemi, P.V. Ruuskanen, S.S. Räsänen, hep-ph/0506049 


\section{Full Hydrodynamic Flow}

Bjorken scaling + Transverse expansion after integration over $m_{T}$

$$
\begin{aligned}
& \left(\frac{d N_{i}}{d y}\right)_{y=0}=\frac{g}{\pi} \int_{\sigma} r d r \tau_{F}(r) \\
& \left\{\cosh \left(y_{T}\right)-\left(\frac{\partial \tau_{F}}{\partial r}\right) \sinh \left(y_{T}\right)\right\} m_{i}^{2} T K_{2}\left(\frac{m_{i}}{T}\right)
\end{aligned}
$$

Consequence : $\frac{d N_{i} / d y}{d N_{j} / d y}=\frac{N_{i}^{0}}{N_{j}^{0}}$

Effects of hydrodynamic flow cancel out in ratio.

$$
\begin{aligned}
Z & =\exp \left\{V \int \frac{d^{3} p}{(2 \pi)^{3}} e^{-\frac{E}{T}+\frac{\mu}{T}}\right\} \\
& =\sum_{N=0}^{\infty} \frac{V^{N}}{N !} e^{\mu N / T}\left[\int \frac{d^{3} p}{(2 \pi)^{3}} e^{-\frac{E}{T}}\right]^{N}
\end{aligned}
$$

with excluded volume corrections

$$
\begin{aligned}
Z \rightarrow & \sum_{N=0}^{\infty} \frac{\left(V-V_{0} N\right)^{N}}{N !} e^{\mu N / T} \\
& {\left[\int \frac{d^{3} p}{(2 \pi)^{3}} e^{-\frac{E}{T}}\right]^{N} \theta\left(V-V_{0} N\right) }
\end{aligned}
$$




\section{Excluded Volume Corrections.}

It is more convenient to consider these corrections in the pressure ensemble:

$$
\begin{aligned}
& Z_{p} \equiv \int_{0}^{\infty} d V e^{-P V / T} \sum_{N=0}^{\infty} \frac{V^{N}}{N !} e^{\mu N / T}\left[\int \frac{d^{3} p}{(2 \pi)^{3}} e^{-\frac{E}{T}}\right]^{N} \\
& Z_{p} \rightarrow \sum_{N=0}^{\infty} \int_{0}^{\infty} d V e^{-P V / T} \\
& \frac{\left(V-V_{0} N\right)^{N}}{N !} e^{\mu N / T} \\
& {\left[\int \frac{d^{3} p}{(2 \pi)^{3}} e^{-\frac{E}{T}}\right]^{N} \theta\left(V-V_{0} N\right) }
\end{aligned}
$$

introduce $x \equiv V-V_{0} N$.

$$
\begin{aligned}
Z_{p}= & \sum_{N=0}^{\infty} \int_{0}^{\infty} d x e^{-P x / T} \\
& \frac{x^{N}}{N !} e^{-P V_{0} N / T} e^{\mu N / T} \\
& {\left[\int \frac{d^{3} p}{(2 \pi)^{3}} e^{-\frac{E}{T}}\right]^{N} }
\end{aligned}
$$

a new variable $\bar{\mu} \equiv \mu-P V_{0}$ 


\section{Excluded Volume Corrections.}

$$
\begin{aligned}
Z_{p} \rightarrow & \sum_{N=0}^{\infty} \int_{0}^{\infty} d x e^{-P x / T} \\
& \frac{x^{N}}{N !} e^{\bar{\mu} N / T}\left[\int \frac{d^{3} p}{(2 \pi)^{3}} e^{-\frac{E}{T}}\right]^{N}
\end{aligned}
$$

which is the original partition function with the replacement

$$
\bar{\mu}=\mu-P V_{0}
$$

\section{Excluded Volume Corrections.}

The particle number density now becomes:

$$
\begin{aligned}
n & =\frac{T}{V} \frac{\partial}{\partial \mu} \ln Z \\
& =\frac{T}{V} \frac{\partial \bar{\mu}}{\partial \mu} \frac{\partial}{\partial \bar{\mu}} \ln Z \\
& =\frac{\partial \bar{\mu}}{\partial \mu} n_{0} \\
& =\left[1-V_{0} n\right] n_{0} \\
n & =\frac{n_{0}}{1+V_{0} n_{0}}
\end{aligned}
$$

\section{Effects Cancel Out in Ratios.}

J.C., K. Redlich, H. Satz, E. Suhonen, ZfP C33, 151, (1986)

D.H. Rischke, M.I. Gorenstein, H. Stöcker, W. Greiner, ZfP C51, 485 (1991). 


\section{Exact Strangeness Conservation.}

For a small system at low temperatures ( $T \approx 50 \mathrm{MeV})$, e.g. at GSI canonical corrections are necessary. Instead of

$$
N_{K} \approx \exp -M_{K} / T
$$

one gets

$$
N_{K} \approx \exp -2 M_{K} / T
$$

Extra suppression is due to strangeness conservation and lack of a large heat bath. This correction disappears quickly at higher energies and is already small at AGS energies.

\section{Exact Strangeness Conservation.}

Insert a Kronecker delta in the trace:

$$
Z=\operatorname{Tr} e^{-\frac{H}{T}+\frac{\mu N}{T}}
$$

$$
\begin{aligned}
& \sum_{i} n_{i}(S=1)+2 \sum_{j} n_{j}(S=2)+3 \sum_{k} n_{k}(3)= \\
& \sum_{i} \bar{n}_{i}(S=-1)+2 \sum_{j} \bar{n}_{j}(S=-2)+3 \sum_{k} \bar{n}_{k}(S=-3)
\end{aligned}
$$

and rewrite it as

$$
\begin{aligned}
& \delta\left(\sum_{i} n_{i}(S=1)+\ldots, \sum_{i} \bar{n}_{i}(S=-1)+\ldots .\right) \\
& =\frac{1}{2 \pi} \int_{0}^{2 \pi} d \phi \\
& \exp \left(i \phi \sum_{i} n_{i}(S=1)+. .-i \phi \sum_{i} \bar{n}_{i}(S=-1)\right)
\end{aligned}
$$




\section{Exact Strangeness Conservation.}

$$
\begin{gathered}
Z=\frac{1}{2 \pi} \int_{0}^{2 \pi} \begin{aligned}
d \phi \exp & \left\{Z_{1} e^{i \phi}+Z_{-1} e^{-i \phi}\right. \\
+ & Z_{2} e^{2 i \phi}+Z_{-2} e^{-2 i \phi} \\
+ & \left.Z_{3} e^{3 i \phi}+Z_{-3} e^{-3 i \phi}\right\}
\end{aligned} \\
=\frac{1}{2 \pi} \int_{0}^{2 \pi} d \phi \exp \left\{\sqrt{Z_{1} Z_{-1}}\left[\sqrt{\frac{Z_{1}}{Z_{-1}}} e^{i \phi}+\sqrt{\frac{Z_{-1}}{Z_{1}}} e^{-i \phi}\right]\right. \\
+\quad \sqrt{Z_{2} Z_{-2}}\left[\sqrt{\frac{Z_{2}}{Z_{-2}}} e^{2 i \phi}+\sqrt{\frac{Z_{-2}}{Z_{2}}} e^{-2 i \phi}\right] \\
\left.+\quad \sqrt{Z_{3} Z_{-3}}\left[\sqrt{\frac{Z_{3}}{Z_{-3}}} e^{3 i \phi}+\sqrt{\frac{Z_{-3}}{Z_{3}}} e^{-3 i \phi}\right]\right\}
\end{gathered}
$$

$Z_{1}$ : sum of all barticles with stranaeness 1. e.a. $K^{+}$

\section{Exact Strangeness Conservation.}

Use

$$
\exp \left\{\frac{x}{2}\left(t+\frac{1}{t}\right)\right\}=\sum_{n=-\infty}^{\infty} I_{m}(x) t^{m}
$$

to obtain

$$
\begin{aligned}
Z= & \frac{1}{2 \pi} \int_{0}^{2 \pi} e^{3 i m \phi+2 i n \phi+i p \phi} \\
& \sum_{\substack{p=-\infty \\
y_{1}^{p} y_{2}^{n} y_{3}^{m}}}^{\infty} I_{p}\left(x_{1}\right) \sum_{n=-\infty}^{\infty} I_{n}\left(x_{2}\right) \sum_{m=-\infty}^{\infty} I_{m}\left(x_{3}\right)
\end{aligned}
$$

where

$$
y_{i}=\sqrt{\frac{Z_{i}}{Z_{-i}}} \quad x_{i}=2 \sqrt{Z_{i} Z_{-i}}
$$




\section{Exact Strangeness Conservation.}

$$
\begin{gathered}
Z=\sum_{m=-\infty}^{\infty} \sum_{n=-\infty}^{\infty} I_{3 m+2 n}\left(x_{1}\right) y_{1}^{-3 m-2 n} \\
I_{n}\left(x_{2}\right) y_{2}^{n} I_{m}\left(x_{3}\right) y_{3}^{m}
\end{gathered}
$$

\section{Exact Strangeness Conservation.}

$$
\begin{aligned}
Z & =\frac{1}{2 \pi} \int_{0}^{2 \pi} d \phi \exp \left\{Z_{1} e^{i \phi}+Z_{-1} e^{-i \phi}\right\} \\
& =\frac{1}{2 \pi} \int_{0}^{2 \pi} d \phi \exp \left\{\sqrt{Z_{1} Z_{-1}}\left[\sqrt{\frac{Z_{1}}{Z_{-1}}} e^{i \phi}+\sqrt{\frac{Z_{-1}}{Z_{1}}} e^{-i \phi}\right]\right\}
\end{aligned}
$$

$Z_{1}$ : sum of all particles with strangeness 1 , e.g. $K^{+}$ $Z_{-1}$ : sum of all particles with strangeness -1 , e.g. $\Lambda$ 


\section{Exact Strangeness Conservation.}

Use

$$
\exp \left\{\frac{x}{2}\left(t+\frac{1}{t}\right)\right\}=\sum_{n=-\infty}^{\infty} I_{m}(x) t^{m}
$$

to obtain

$$
Z=\frac{1}{2 \pi} \int_{0}^{2 \pi} e^{i p \phi} \sum_{p=-\infty}^{\infty} I_{p}\left(x_{1}\right) y_{1}^{p}
$$

where

$$
y_{1}=\sqrt{\frac{Z_{1}}{Z_{-1}}} \quad x_{1}=2 \sqrt{Z_{1} Z_{-1}}
$$

$$
Z=I_{0}\left(x_{1}\right)
$$

\section{Exact Strangeness Conservation.}

In more detail, e.g. the multiplicity of $K^{+}$

$$
N_{K^{+}}=\left.\frac{T}{Z} \frac{\partial I_{0}\left(x_{1}\right)}{\partial \mu_{K^{+}}}\right|_{\mu_{K^{+}}=0}
$$

Use

$$
\frac{d}{d z} I_{0}(z)=I_{1}(z)
$$




\section{Exact Strangeness Conservation.}

$$
\begin{aligned}
N_{K^{+}} & =\frac{T}{Z} \frac{\partial}{\partial \mu_{K^{+}}} I_{0}\left(x_{1}\right) \\
& =\frac{T}{I_{0}\left(x_{1}\right)} I_{1}\left(x_{1}\right) \frac{\partial x_{1}}{\partial \mu_{K^{+}}} \\
& =\frac{T}{I_{0}\left(x_{1}\right)} I_{1}\left(x_{1}\right) \frac{\partial 2 \sqrt{Z_{1} Z_{-1}}}{\partial \mu_{K^{+}}} \\
& =\frac{I_{1}\left(x_{1}\right)}{I_{0}\left(x_{1}\right)} \sqrt{\frac{Z_{-1}}{Z_{1}}} N_{K^{+}}^{0}
\end{aligned}
$$

where $N_{K^{+}}^{0}$ refers to the "unmodified" kaon multiplicity.

\section{Exact Strangeness Conservation.}

In the small volume limit this becomes

$$
\lim _{z \rightarrow 0} I_{0}(z)=1
$$

and

$$
\begin{aligned}
& \lim _{z \rightarrow 0} I_{1}(z)=\frac{z}{2} \\
\lim _{V \rightarrow 0}= & N_{K^{+}}^{0} Z_{-1} \\
\lim = & N_{K^{+}}^{0} Z_{-1} \\
= & N_{K^{+}}^{0}\left[N_{K^{-}}^{0}+N_{\Lambda}^{0}+\cdots\right]
\end{aligned}
$$

i.e., the particle multiplicity is

- proportional to $V^{2}$, and not $V^{1}$. 


\section{Exact Strangeness Conservation.}

In the small volume limit this becomes

$$
\lim _{z \rightarrow 0} I_{0}(z)=1
$$

and

$$
\begin{aligned}
& \lim _{z \rightarrow 0} I_{1}(z)=\frac{z}{2} \\
\lim _{V \rightarrow 0}= & N_{K^{+}}^{0} Z_{-1} \\
\lim = & N_{K^{+}}^{0} Z_{-1} \\
= & N_{K^{+}}^{0}\left[N_{K^{-}}^{0}+N_{\Lambda}^{0}+\cdots\right]
\end{aligned}
$$

i.e., the particle multiplicity is

- proportional to $V^{2}$, and not $V^{1}$.

- proportional to $\exp \left(-2 m_{K} / T\right)$ or to $\exp \left(-\left(m_{K}+m_{\wedge}\right) / T\right)$ and not simply $\exp \left(-m_{K} / T\right)$, i.e. there is additional suppression of strange particles.

\section{Exact Strangeness Conservation}

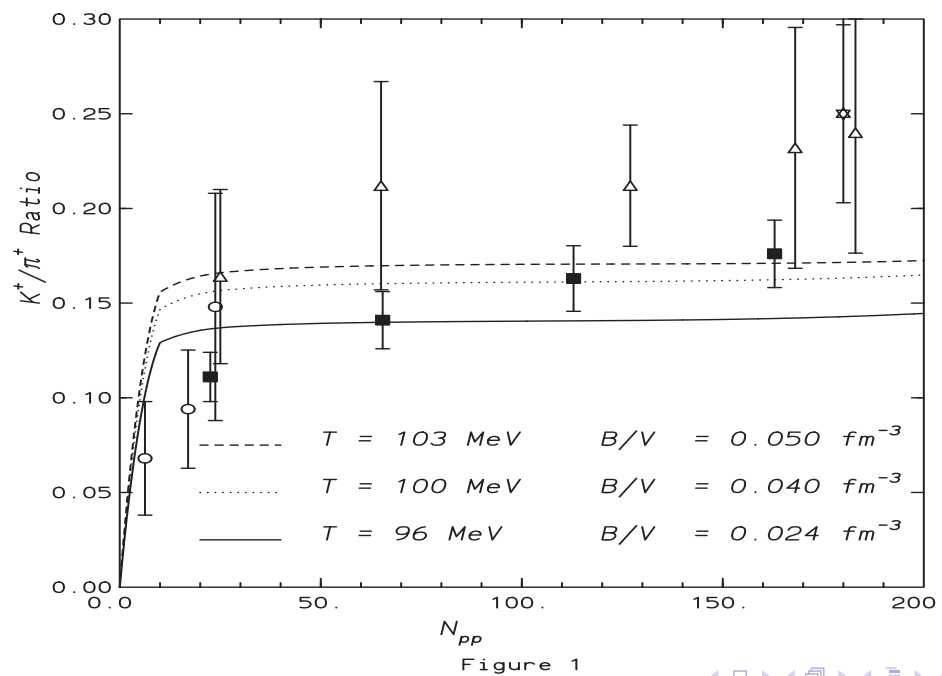




\section{Transverse Momentum in the Thermal Model}

$$
\begin{gathered}
E_{i} \frac{d N_{i}}{d^{3} p}=\frac{g_{i}}{(2 \pi)^{3}} V E_{i} \exp \left(-\frac{E_{i}}{T}+\frac{\mu_{i}}{T}\right) \\
\frac{d N_{i}}{d y m_{T} d m_{T}}=\frac{g_{i}}{(2 \pi)^{2}} V m_{T} \cosh y e^{-\frac{m_{T}}{T} \cosh y+\frac{\mu_{i}}{T}} \\
\frac{d N_{i}}{m_{T} d m_{T}}=\frac{g_{i}}{2 \pi^{2}} V m_{T} K_{1}\left(\frac{m_{T}}{T}\right) e^{\frac{\mu_{i}}{T}}
\end{gathered}
$$

For large values of $m_{T}$ :

$$
\frac{d N_{i}}{d m_{T}}=\frac{g_{i}}{2 \pi^{2}} V m_{T}^{3 / 2} \exp \left(-\frac{m_{T}}{T}\right) \sqrt{\frac{T \pi}{2}} e^{\frac{\mu_{i}}{T}}
$$

$$
\text { SCALING in } m_{T}
$$

$$
\begin{gathered}
\frac{d N_{i}}{d y m_{T} d m_{T}}=\frac{g_{i}}{(2 \pi)^{2}} V m_{T} \cosh y e^{-\frac{m_{T}}{T} \cosh y+\frac{\mu_{i}}{T}} \\
\frac{d N_{i}}{d y}=\frac{g_{i} V}{2 \pi^{2}}\left[\frac{2 T^{3}}{\cosh ^{2} y}+\frac{2 m T^{2}}{\cosh y}+m^{2} T\right] e^{\frac{\mu_{i}}{T}} \\
e^{-\frac{m}{T} \cosh y}
\end{gathered}
$$

Narrow Distribution in Rapidity Approximately Gaussian 


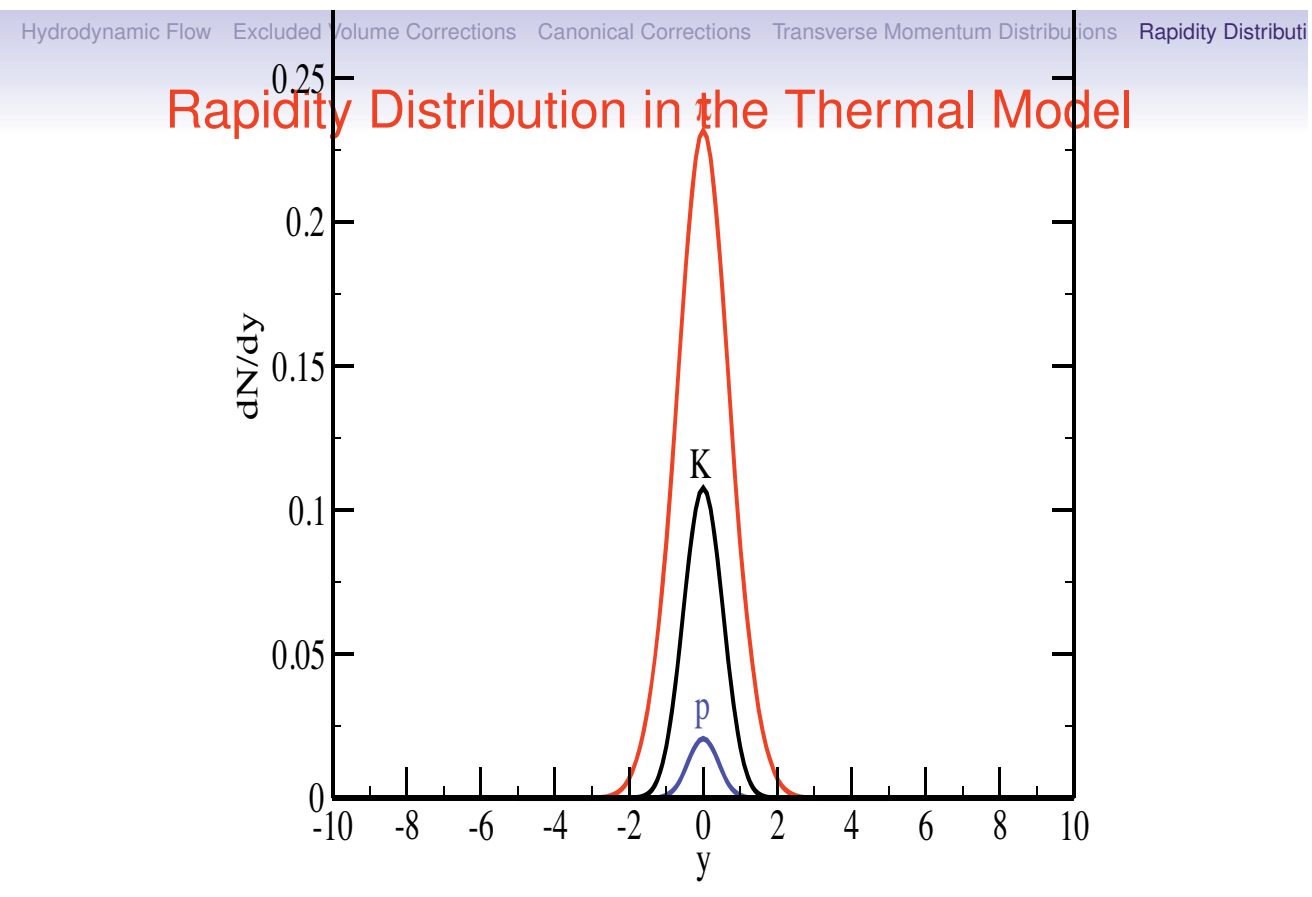

\section{Superposition of Fireballs.}

$$
\frac{d N_{i}}{d y}=\int_{-Y}^{Y} d Y_{F B} \rho\left(Y_{F B}\right) \frac{d N_{i}^{0}}{d y}\left(y-Y_{F B}\right)
$$

where

$$
\begin{aligned}
\frac{d N_{i}^{0}}{d y}= & \frac{g_{i} V}{2 \pi^{2}}\left[\frac{2 T^{3}}{\cosh ^{2}\left(y-Y_{F B}\right)}+\frac{2 m T^{2}}{\cosh \left(y-Y_{F B}\right)}+m^{2} T\right] e^{\frac{\mu_{i}}{T}} \\
& e^{-\frac{m}{T} \cosh \left(y-Y_{F B}\right)}
\end{aligned}
$$




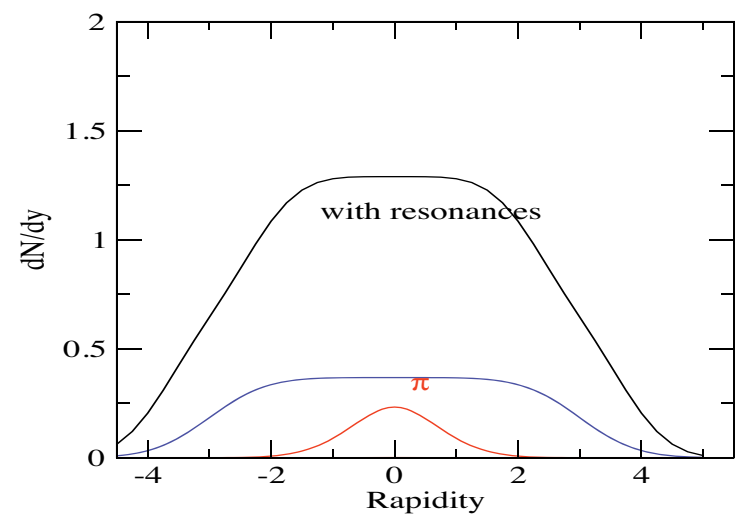

$$
\begin{gathered}
n_{i}=\int_{-\infty}^{\infty} d y \int_{-Y}^{Y} d Y_{F B} \rho\left(Y_{F B}\right) \frac{d n_{i}^{0}}{d y}\left(y-Y_{F B}\right) \\
n_{i}=\int_{-Y}^{Y} d Y_{F B} \rho\left(Y_{F B}\right) \int_{-\infty}^{\infty} d y \frac{d n_{i}^{0}}{d y}\left(y-Y_{F B}\right) \\
n_{i}=g_{i} V \frac{1}{2 \pi^{2}} T m_{i}^{2} K_{2}\left(\frac{m_{i}}{T}\right) e^{\frac{\mu_{i}}{T}} \int_{-Y}^{Y} d Y_{F B} \rho\left(Y_{F B}\right)
\end{gathered}
$$

Equivalent to changing the volume $V$. 


\section{Superposition of Fireballs.}

$$
\begin{gathered}
\frac{n_{i}}{n_{j}}=\frac{\int_{-\infty}^{\infty} d y \int_{-Y}^{Y} d Y_{F B} \rho\left(Y_{F B}\right) \frac{d n_{i}^{0}}{d y}\left(y-Y_{F B}\right)}{\int_{-\infty}^{\infty} d y \int_{-Y}^{Y} d Y_{F B} \rho\left(Y_{F B}\right) \frac{d n_{i}^{0}}{d y}\left(y-Y_{F B}\right)} \\
\frac{n_{i}}{n_{j}}=\frac{n_{i}^{0}}{n_{j}^{0}} \frac{\int_{-Y}^{Y} d Y_{F B} \rho\left(Y_{F B}\right)}{\int_{-Y}^{Y} d Y_{F B} \rho\left(Y_{F B}\right)} \\
\frac{n_{i}}{n_{j}}=\frac{n_{i}^{0}}{n_{j}^{0}}=\frac{m_{i}^{2}}{m_{j}^{2}} \frac{K_{2}\left(m_{i} / T\right)}{K_{2}\left(m_{j} / T\right)} e^{\left(\mu_{i}-\mu_{j}\right) / T}
\end{gathered}
$$

Effects Cancel Out in Ratios. 